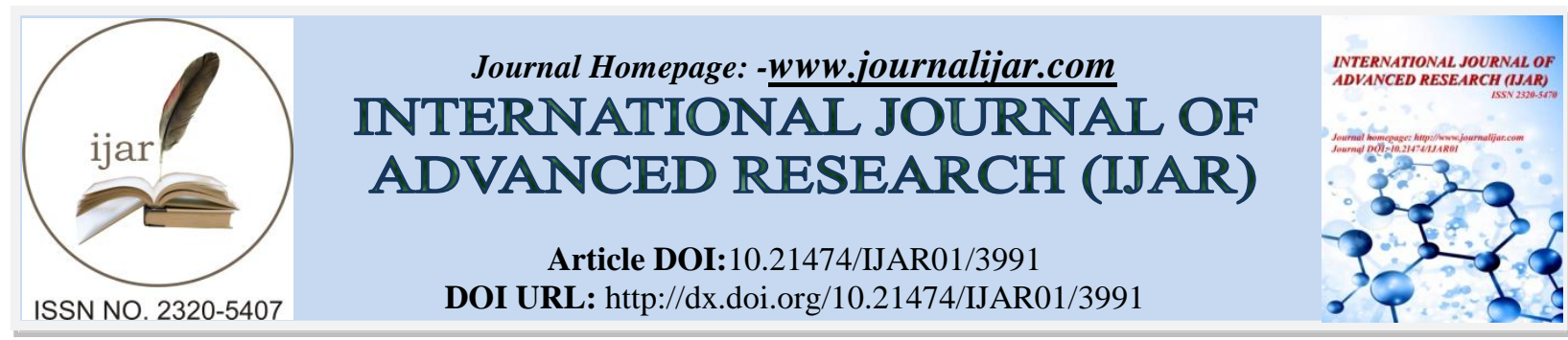

RESEARCH ARTICLE

\title{
EFFECT OF ABHRAK BHASMA ON THE PHYSIOLOGY AND BEHAVIOUR OF DROSOPHILA MELANOGASTER.
}

\section{Rambhadur P. Subedi ${ }^{1}$, Rekha R. Vartak ${ }^{2}$ and Purushottam G. Kale ${ }^{1^{*}}$}

1. Department of Biological Sciences, RamniranjanJhunjhunwala College, Mumbai, Maharashtra, India.

2. Biology Olympiad Cell, HomiBhabha Centre for Science Education-Tata Institute of Fundamental Research, Mumbai, Maharashtra, India.

\section{Manuscript Info}

Manuscript History

Received: 20 February 2017

Final Accepted: 23 March 2017

Published: April 2017

Key words:-

Ayurveda, Nanoparticle, RING assay
(.........................

\begin{abstract}
Bhasma, claimed to be biologically produced nanoparticles, are Ayurvedic preparations used in treating a variety of ailments. Abhrak bhasma is derived from mica mineral through repeated incineration after mixing with certain plant materials. The effect of abhrak bhasma on Drosophila was studied in terms of longevity and effect on body weight, heat tolerance as well as behavioural modifications. The behavioural modifications were investigated using locomotor assay and pupation height for larvae and Retro-Iterative Negative Geotaxis assay (RING assay) for adults. There is paucity of literature that describes the effect of abhrak bhasma in general and in insect model system in particular. Drosophila has been extensively used as a model system for a wide range of studies. Owing to the short life cycle and availability of vast scientific data, diverse factors that are affected by any treatment can be studied comprehensively in Drosophila. This is the first report on using Drosophila melanogaster to demonstrate the effect of abhrak bhasma. In this study, abhrak bhasma has been found to increase the median lifespan of flies, to strengthen the locomotory abilities and to increase the mating efficiency. Further studies are however needed to understand the exact mechanism of these responses.
\end{abstract}

Copy Right, IJAR, 2017,. All rights reserved.

\section{Introduction:-}

Bhasma are the products of calcination in which metallic or mineral preparations are treated with herbal juices or decoctions and incinerated, according to puta system of ayurvedal, ${ }^{1,2}$ Bhasma are considered to be a type of nanomedicine ${ }^{3}$ and free from toxicity at therapeutic doses ${ }^{4}$. They have traditionally been used as medicine in treating a vast range of conditions like inflammatory diseases, diabetes, osteoarthritis, infertility, erectile dysfunction, ulcers etc. $^{1,5,6}$. The type of bhasma used depends primarily on the type of the disease under consideration.

Abhrak bhasma $(\mathrm{AB})$ is prepared from biotite, the brown-black mineral mica and about 72 types of herbs that are incinerated repeatedly in several cycles, called 'puta' in ayurvedic pharmacological system ${ }^{2,3}$. The juice, sap and decoctions of these herbs are mixed with the mineral mica and subjected to incineration for specified number of puta or cycles to obtain the final bhasma product. Typically, to prepare different grades of abhrak bhasma, the

Corresponding Author:-Purushottam G. Kale

Address:-Department of Biological Sciences, RamniranjanJhunjhunwala College, Mumbai, 
putaprocess is repeated 7-1000 times. Thus, shataputi abhrak bhasma goes through 100 putas while shahastraputi abhrak bhasma, which is considered to be of the finest quality, involves 1000 putas.

$\mathrm{AB}$ is used in the treatment of a wide range of diseases that include hepatitis, tuberculosis, asthma, plague, gastritis, skin diseases etc. ${ }^{7}$ AB is also considered as an excellent cell regenerator and as a nervine tonic, thus used in many rejuvenation formulations ${ }^{8}$. Recently, the efficacy of $\mathrm{AB}$ on the proliferation of germinal epithelium in rats exposed to heat stress has been established ${ }^{9}$.

An increasing number of people are seeking alternative treatments in Ayurveda and other forms of natural medicines as these are claimed to have minimal side-effects compared to allopathic medicines and work to eradicate the root cause of the disorder instead of seeking symptomatic relief. There is a serious lack of studies to evaluate the effectiveness of these ayurvedic preparations using different model systems. Exploration of NCBI PMC database resulted in only 260 hits on bhasma, 12 hits on abhrak bhasma, only 4 hits on bhasma and Drosophila whereas 0 hit on abhrak bhasma and Drosophila (as of 03/006/2016). Testing the efficacy of such preparations directly on humans and other mammals is cumbersome and one has to deal with the ethical concerns. Drosophila melanogaster has been widely used as a model organism in genetics, physiology, ageing, drug research, etc., owing to its short life cycle, ease in culturing and ease of doing experimentation. Since diverse factors that affect life history traits can be easily studied in Drosophila and abundance of scientific data available for Drosophila, it can be an excellent model system to test ayurvedic preparations and understand the mechanism of action in detail.

The present study explores such possibility of testing the efficacy of $\mathrm{AB}$ in $D$. melanogaster with a view to employing it to monitor demography of this insect. This preliminary work is focused mainly on comprehending physiological and behavioural effects of AB in Drosophila. This work may also be extended to other chemical species, for understanding physiological and behavioural effects in Drosophila and thus, can be employed in regulating demography of insects.

\section{Material andMethods:-}

Drosophila Husbandry:-

Drosophila melanogaster (Canton S strain) fly cultures were maintained at $26 \pm 1^{\circ} \mathrm{C}$ with $12 \mathrm{hr}$ light and $12 \mathrm{hr}$ dark cycle. Control flies were maintained in the corn-flour-yeast-agar medium consisting of corn flour, dextrose, sucrose, agar-agar and yeast. Sahastraputi AB was procured from Shree Dhootapapeshwar Ltd. and the product of a single batch was used for all the experiments. For preparing $\mathrm{AB}$ test media, $\mathrm{AB}$ at specific concentration was mixed in the same recipe as used for control flies, before solidification of the media. Drosophila flies were transferred to the respective fresh media bottles every 6-7 days in order to maintain the specific age groups.

\section{AB tolerance Assay:-}

Control flies were allowed to lay eggs on fresh medium laid in a series of bottles for 12 hours. After 24 hours, $20 \%$ sucrose was put into these bottles and swirled. This sucrose solution containing the eggs and $1^{\text {st }}$ instar larvae was collected in Petri dishes. Using Olympus Magnus stereo-microscope (model number MS13) the $1^{\text {st }}$ instar larvae were separated, washed with distilled water and segregated in triplicate of 4 sets, each with 25 larvae. The larvae were transferred into respective media containing $0 \%$ (control), $0.1 \%, 0.5 \%$ and $2 \%$ abhrak bhasma. These larvae were allowed to grow till adulthood. Numbers of larvae surviving up to adulthood were counted and the result was expressed as the mean number of survivors in each of these $\mathrm{AB}$ concentrations.

\section{Pupation height and time required for development from egg to adult:-}

In separate tubes having the culture medium containing varying concentrations of abhrak bhasma $(0 \%, 0.1 \%, 0.5 \%$ and $2.0 \%)$ the first instar larvae (30 each) were transferred to each tube in triplicates. All tubes were maintained under identical conditions like temperature and light-dark cycle, till the larvae were transformed into pupae. The average height at which each of the larvae pupates in a tube was recorded.

It was learnt in pilot experiments that pupation was achieved in most of the $\mathrm{AB}$ concentrations in 168 hours. Thus, the pupae were collected from media containing different concentrations of $\mathrm{AB}$, immediately after 168 hours. The number of larvae, if any, yet to pupate, was counted for the media containing specific $\mathrm{AB}$ concentrations. The time of emergence of the flies from the pupae was then recorded for each group. 


\section{Body weight Analysis:-}

In yet another series of experiments, the third instar larvae, about 100 , from each of the control and $0.1 \% \mathrm{AB}$ media were removed and washed thoroughly with distilled water. The larvae were blotted with tissue paper to remove the excess of water. The individual larva, picked up with a fine brush, was weighed using the weighing machine (Citizen scale, model CX220) with a sensitivity of $\pm 0.1 \mathrm{mg}$. The adult flies from the control and $0.1 \% \mathrm{AB}$ media were collected within 2 hours of eclosion (emergence of an adult Drosophila from a pupal case) by killing instantly with ethyl acetate. In total, 160 adults (80male, 80female) were weighed from each food regime and the mean weight with the standard error was calculated.

\section{Study of life span:-}

In one more series of experiments, the flies were allowed to lay eggs for 6 hours in bottles containing media with $0 \%$ and $0.1 \% \mathrm{AB}$. About 100 of the newly emerged adult flies from the control and $0.1 \% \mathrm{AB}$ fed larvae, in a $1: 1$ male to female ratio, were transferred into fresh culture bottles containing $0 \%$ and $0.1 \% \mathrm{AB}$, in four replicates. These adults were maintained at $26^{\circ} \mathrm{C}$, with 12 hours light and 12 hours dark cycle. On every $7^{\text {th }}$ day, the adults were transferred to new culture bottles and the number of dead organisms was counted. This was repeated till all the flies died.

\section{Behaviour study:-}

Behavioural studies on Drosophila larvae and adults were carried out as described by Nicholas et al. ${ }^{10}$. This study was carried out in three parts as follows:

\section{Larval Locomotor Assay:-}

The flies were allowed to lay eggs for 4 hours in the bottles containing culture media with $0 \%$ (control) and $0.1 \%$ AB.After 5 days, $3^{\text {rd }}$ instar larvae were seen crawling onto the walls of culture bottles. These $3^{\text {rd }}$ instar larvae were collected using a brush and washed thoroughly with distilled water before proceeding to the further experiments involving two assays as follows.

\section{The total distance travelled / Crawling Assay:-}

Experiments were performed using Petri dishes with the diameter of $8.0 \mathrm{~cm}$, laid with $2 \%$ agarose and the $3^{\text {rd }}$ instar larvae transferred to the centre. The dishes were kept on a graph paper with $1 \mathrm{~mm}^{2}$ grid lines (Figure 1). Video recording was carried out using Honor 4C device with a 13-megapixel camera at $\operatorname{HD} 720 \mathrm{P}$ (16:9, stereo) setting. The number of grid lines crossed in 1 minute by each larva was carefully counted from the recorded video. Three sets of readings were taken for each larva and 30 larvae were sampled from each of the concentrations of $\mathrm{AB}$.

\section{The body wall contractions / peristalsis Assay:-}

A single larva at a time from the control and $0.1 \% \mathrm{AB}$ feeding regime was transferred to the cavity of a cavity well microscope slide containing distilled water. The number of body contractions, from one end to another (usually posterior to anterior), was counted for one minute under the Olympus Magnus dissection microscope. Three sets of readings were taken for the single larva and 30 such larvae from each food regime were examined for this assay.

\section{Rapid Iterative Negative Geotaxis Assay / RING Assay:-}

This assay described by Garganoet al. ${ }^{11}$ was used with certain modifications. Without using any anesthetics, 25 males and 25 females of two days old, were sorted into different RING assay tubes using a self-made aspirator (RamAspire) for transfer of the flies (Figure 2). The flies were allowed to acclimatize in these assay tubes for 1 minute. The camera system (Honor 4C device with 13megapixel lens) was set up horizontally in relation to the RING assay apparatus. Video capturing with HD 720p setting was done from the moment the flies were transferred to the assay tubes. After 1 minute, the apparatus was tapped thrice and the recording was continued. This procedure was repeated 3-4 times with a resting period of 30 seconds between every set of readings. The video was later analysed considering zero time when all the flies were at the bottom of the apparatus. Screenshots were taken at 0 seconds and 2 seconds time points. These images were then used for the measurement of mean distance climbed by each Drosophila in ImageJ software. Mean height climbed by the flies raised from control as well as $0.1 \% \mathrm{AB}$ fed larvae was calculated and plotted.

Mating Assay:-

Newly emerged male and female flies from control as well as $0.1 \% \mathrm{AB}$ feeding regime of less than 2 hours in age, were collected into separate media tubes. Males were segregated, one adult per culture tube, while females were 
kept in a group of 5-6 individuals in separate culture tubes and kept in isolation for 72 hours. Mating assays between male and female of the same food regime were then carried out in self-designed RASU mating wells (Figure 3). Video capture, using the same device as mentioned above, was begun when a single pair of male and female was transferred to a RASU mating well. This video was later analysed for courtship period and duration of mating.

\section{Thermo-tolerance Test:-}

One to two days old flies, about 100 adults in triplicates, from control as well as $0.1 \%$ AB fed larvae were given a temperature shock at $37^{\circ} \mathrm{C}, 39^{\circ} \mathrm{C}$ and $40^{\circ} \mathrm{C}$ for 1 hour. The flies from both the feeding regimes kept at $26^{\circ} \mathrm{C}$, were taken as temperature shock controls. The numbers of surviving adults were counted after 24 hours of treatment. The flies were considered dead when none of the appendages moved after touching with a brush.

\section{Statistical Analysis:-}

Most of the experiments were performed in triplicates using the total number of flies $(\mathrm{N}) \geq 30$. In certain experiments, where three sets were not kept, the sample number of flies $(\mathrm{N})$ was kept greater than 100 for the reliability of the results. The differences in the mean of control versus test flies were analysed using the student's ttest in GraphPad prism software. The null hypothesis was stated as "any differences, discrepancies, or suspiciously outlying results between control and the treated group is purely due to sampling and experimental errors" in all tests. The null hypothesis was rejected when the p-value was less than 0.05 . For comparison of more than two means, one way ANOVA test was applied and post-hoc analysis were carried out using the Tukey HSD test. Deviation in all experimental data was mentioned in the form of standard error and represented as " \pm " values.

\section{Results:-}

\section{Drosophila larva tolerate high dose of AB:-}

D. melanogaster larvae showed tolerance up to $2 \% \mathrm{AB}$ (maximum concentration studied). The differences in survival rate between all groups under study were found statistically insignificant (Figure 4). The number of larvae surviving in control, $0.1 \% \mathrm{AB}, 0.5 \% \mathrm{AB}, 2 \% \mathrm{AB}$ feeding media were $99 \pm 1.3 \%, 97 \pm 1.3 \%, 96 \pm 2.3 \%, 97 \pm 1.3 \%$ respectively (ANOVA p-value $=0.730695)$.

\section{AB delays the larval development and also lowers the pupation height at high concentration:-}

The pupation height was found to decrease with an increase in the concentration of AB. Differences in pupation height of control versus $0.1 \% \mathrm{AB}$ fed larvae were statistically insignificant while it differed significantly for larvae fed on $0.5 \% \mathrm{AB}$ and $2 \% \mathrm{AB}$ as compared to the control larvae (ANOVA p-value $=0.000882$ ) (Figure 5). On the 7th day, after most of the larvae were transformed into pupae, all pupae and remaining larvae, after carefully dissolving the media in distilled water, were counted under the stereomicroscope. While no larvae were seen in control, $0.1 \%$ $\mathrm{AB}$ and $0.5 \% \mathrm{AB}, 2 \% \mathrm{AB}$ still had larvae in 3rd instar stage $(8 \pm 1 \%)$. The increase in development time in a concentration-dependent manner was also evident from the eclosion time difference between the different feeding regimes (Figure 6). The higher dose of $\mathrm{AB}$ of $2 \%$ was found to shift total pupa to adult development time by 20 hrs. (Note: only pupae from all food regimes that pupated at $168 \mathrm{hrs}$ were considered for eclosion time difference)

As $0.1 \% \mathrm{AB}$ fed larvae showed statistically insignificant difference in development time as well as pupation height compared to control, $0.1 \% \mathrm{AB}$ feeding regime was used for the subsequent assays.

AB reduces the body weight of newly emerged Adults:-

Mean body weight of males emerging from larvae maintained on medium without AB was $0.93 \pm 0.03$ mg while that of males emerging from $\mathrm{AB}$ fed larvae was $0.64 \pm 0.02 \mathrm{mg}$. This reduction in body weight was $31 \%$ with a pvalue of $<0.0001$. The body weight of females emerging from larvae in the control medium was $1.27 \pm 0.03 \mathrm{mg}$ while that of $\mathrm{AB}$ fed females was $0.9 \pm 0.03 \mathrm{mg}$. Females from $\mathrm{AB}$ fed larvae had a significant reduction in the body weight by $29 \%$ with p-value $<0.0001$ (Figure 7 ). Body weight of 3rd instar larvae of Control and $0.1 \% \mathrm{AB}$ feeding regime was $1.65 \pm 0.05$ and $1.54 \pm 0.04$ respectively, but, this difference in weight was found to be statistically insignificant (p-value 0.0872).

\section{Increase in median life span (50\% survival) of the adults from $0.1 \% \mathrm{AB}$ fed larvae:-}

The adults from Control and $0.1 \% \mathrm{AB}$ fed larvae were transferred to new respective media bottle on every 7 th day and the numbers of dead organisms were counted after each transfer. The median life span i.e. day by which $50 \%$ of the population survived was calculated from the survivorship curve graph. It was observed that median lifespan of adults from $\mathrm{AB}$ fed larvae increased by $7 \pm 1$ days with compared to control (Figure 8 ). 


\section{Increase in locomotor response due to $\mathrm{AB}$ feeding:-}

The number of $1 \mathrm{~mm}^{2}$ grid crossed per minute in the locomotor assay by control larvae was $24 \pm 2.3$ grid units/minute, while $0.1 \% \mathrm{AB}$ fed larvae crossed $38 \pm 2.6$ grid units/minute (Figure 9a). AB fed larvae crossed a significantly greater number of $1 \mathrm{~mm}^{2}$ grid units as compared to the control larvae ( $\mathrm{p}$-value $=0.0009$ ).

Similar results were obtained for the peristalsis assay where AB fed larvae showed a significantly greater number of peristaltic contractions as compared to control larvae (p-value: 0.0004). Mean peristaltic contractions per minute for control and $0.1 \% \mathrm{AB}$ larvae were $19 \pm 2.8$ and $34 \pm 1.9$, respectively (Figure $9 \mathrm{~b}$ ).

\section{Adults from 0.1\% AB fed larvae show higher climbing Response:-}

RING assay was separately performed for male and female Drosophila with about 25 adults in each group per experiment. Mean height climbed by control males was $4.7 \pm 0.2 \mathrm{~cm} / 2 \mathrm{~s}$ while that of $\mathrm{AB}$ test males was $6.5 \pm 0.2 \mathrm{~cm} / 2 \mathrm{~s}$ (Figure 8). Similarly, mean height climbed by control female fly was $5 \pm 0.3 \mathrm{~cm} / 2$ seconds and by AB test female was $5.9 \pm 0.2 \mathrm{~cm} / 2$ seconds (Figure 10). AB fed males as well as female Drosophila adults climbed significantly more height compared to the control flies with respective p-values of 0.001 and 0.0185 .

\section{AB increases the number of successful larvae produced per copulation minute per mating:-}

Virgin males and females were collected within two hours of eclosion. These adults were allowed to mate after 3 days of isolation. Successful larvae produced were counted after 8-9 days when the larvae were in 2nd to 3rd instar stage. As females can store sperms for up to two weeks after mating ${ }^{12}$, counting of larvae was continued for next two weeks. It was observed that even though flies emerging from $\mathrm{AB}$ fed larvae spend less time in mating (Figure 11a), the number of successful larvae produced per mating per copulation minute was significantly higher than the control flies ( $\mathrm{p}=0.0103$ ) (Figure 11b).

\section{No significant differences in response to the temperature shock:-}

One to two day old adult flies were tested for response to heat shock at $37^{\circ} \mathrm{C}, 39^{\circ} \mathrm{C}$ and $40^{\circ} \mathrm{C}$. Flies from control as well as $0.1 \% \mathrm{AB}$ feeding regime survived the thermal shock at $37^{\circ} \mathrm{C}$ and $39^{\circ} \mathrm{C}$ for 1 hour $(>95 \%$ survival). The thermal shock at $40^{\circ} \mathrm{C}$ for 1 hour had a lethal effect ( $>95 \%$ death) on adults from control as well as $\mathrm{AB}$ fed larvae, however, the flies from both the groups tolerated $40^{\circ} \mathrm{C}$ shock given for 30 minutes (>90\% survival). The flies emerging from larvae fed on $\mathrm{AB}$ showed no statistically significant difference in mortality in response to any of these temperature shocks, when compared to the flies emerging from larvae maintained in control medium $(\mathrm{p}=0.7510)$. The adults that survived $39^{\circ} \mathrm{C}$ shock were further analysed for survivorship pattern. It was observed that the median life span of heat shocked adults from $\mathrm{AB}$ as well as control larvae decreased as compared to their respective non-heat shocked adults. However, the survivorship curve of heat shocked adults from $\mathrm{AB}$ as well as control feeding showed no significant difference in response to $39^{\circ} \mathrm{C}$ temperature stress among themselves (Figure 12).

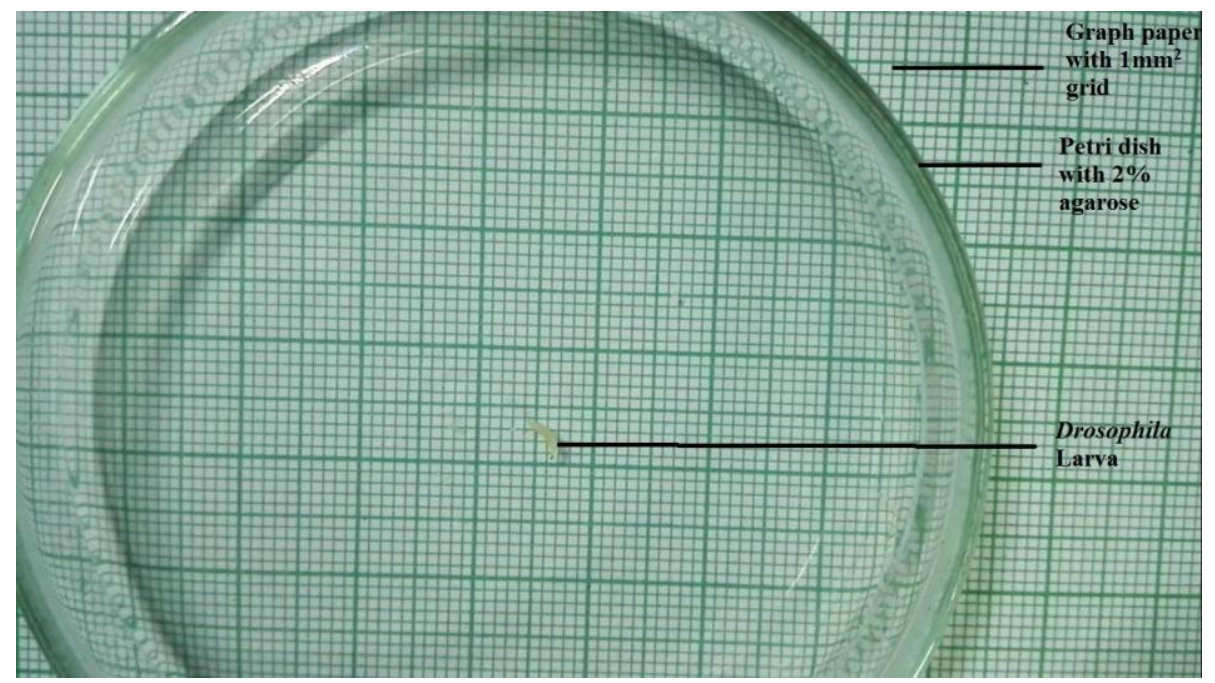

Figure 1:-larval locomotion assay grids.In a transparent Petri dish, $2 \%$ agarose is prepared and put on a graph paper with $1 \mathrm{~mm}^{2}$ grids. A camera system is placed above this unit to record the larval locomotion. 


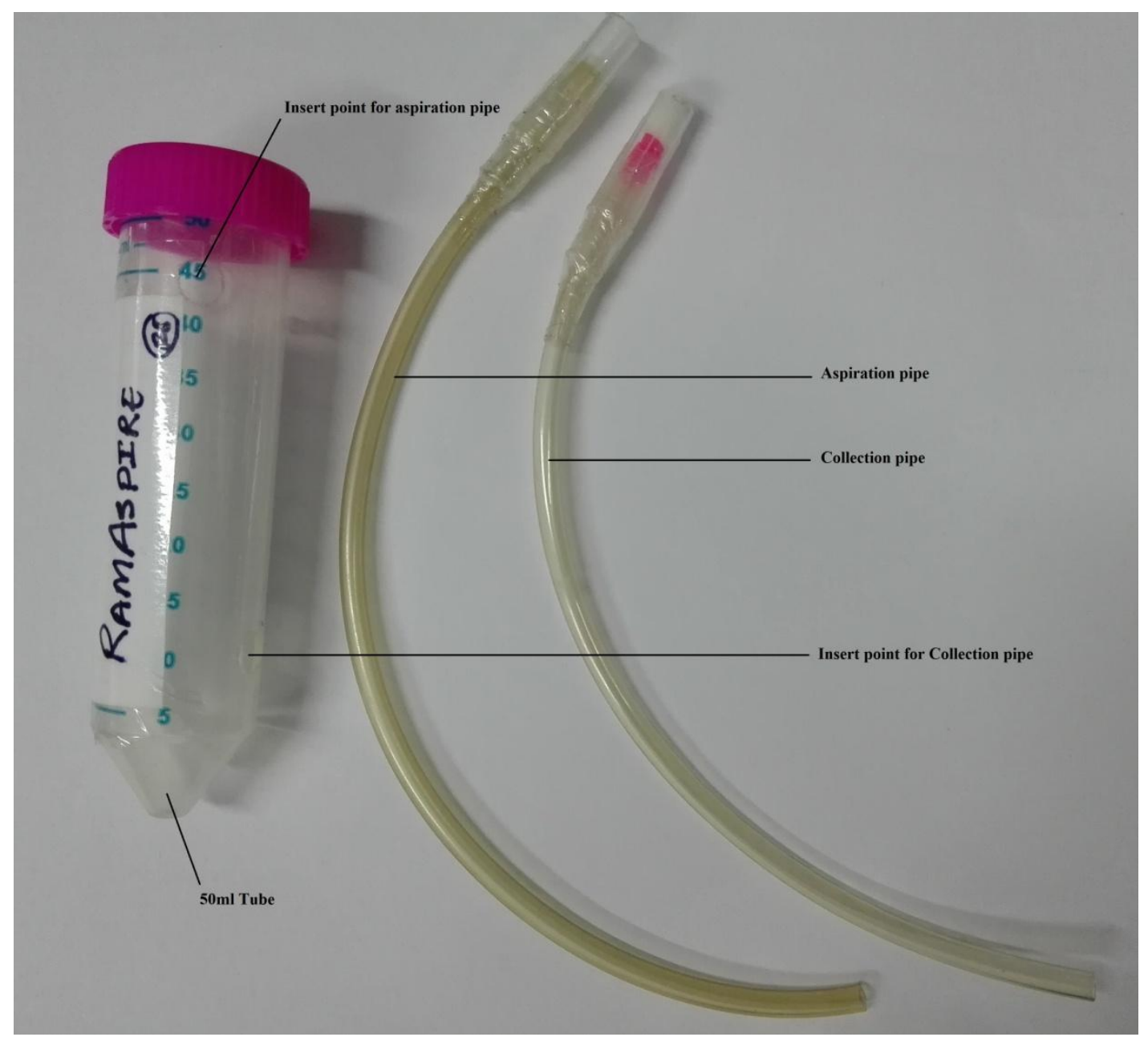

Figure 2:-RamAspire aspirator for fly transfer.It is a simple $50 \mathrm{ml}$ tube with two insert points, one near the top and another near the bottom of the tube. Two similar interchangeable hollow pipes are connected to the tube through these insert points. One pipe acts as aspiration pipe while the other as a collection pipe. Usually, 1-5 Flies are collected and can be transferred to designated place through collecting pipe. For large number sample, the 50ml tube functions as a collecting tube. 


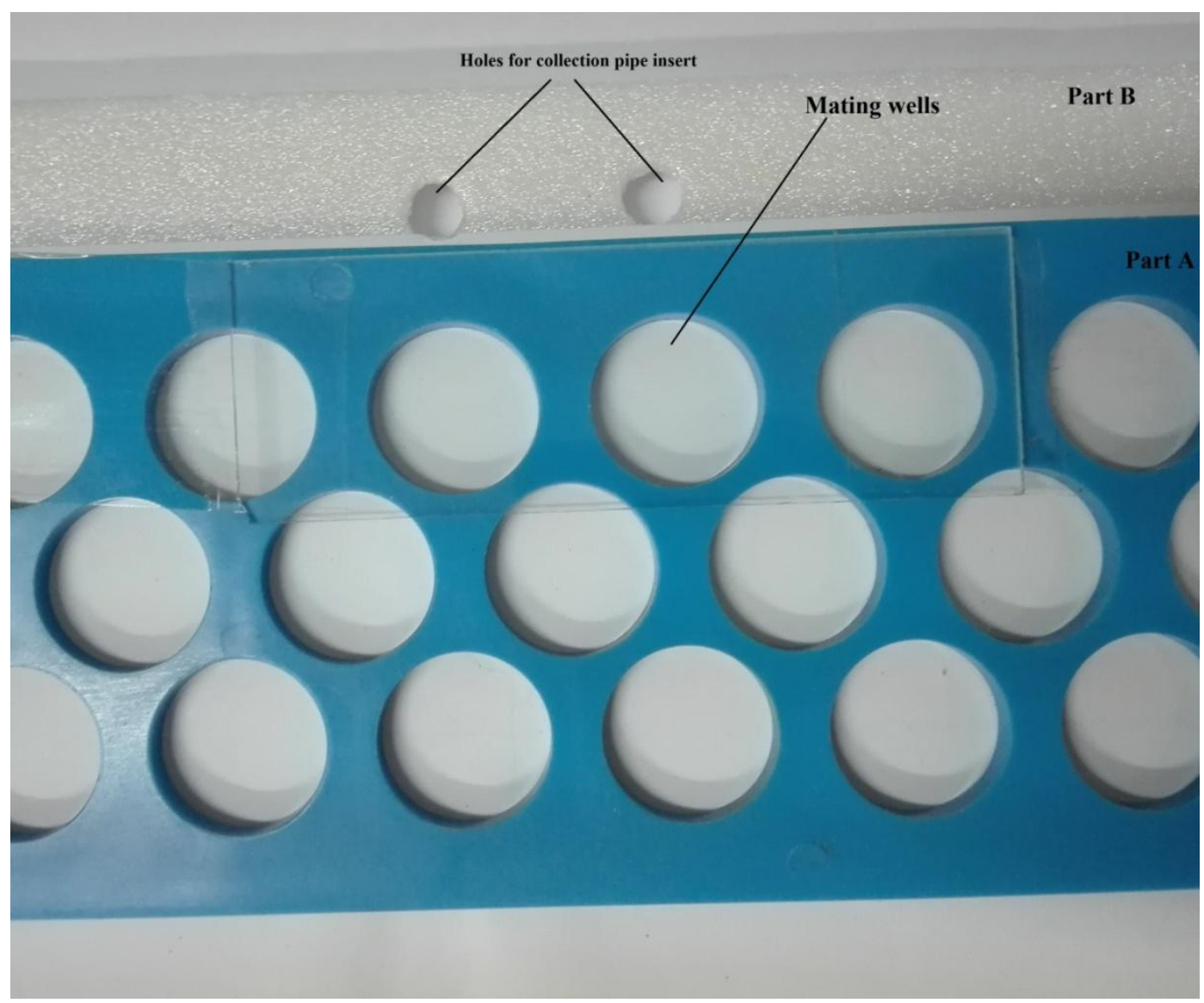

Figure 3:-RASU mating wells.Each RASU mating well is $0.85 \mathrm{~cm}$ in radius and $3 \mathrm{~mm}$ in depth. A small hole is made, through which collecting pipe of RamAspire can be inserted for transferring the flies. When the flies are transferred, part A is made to slide over part B so that mating wells are away from the insert holes. Because of low depth of the wells, all the movements and behaviour of flies can be easily monitored and recorded in video camera without losing the focus on flies.

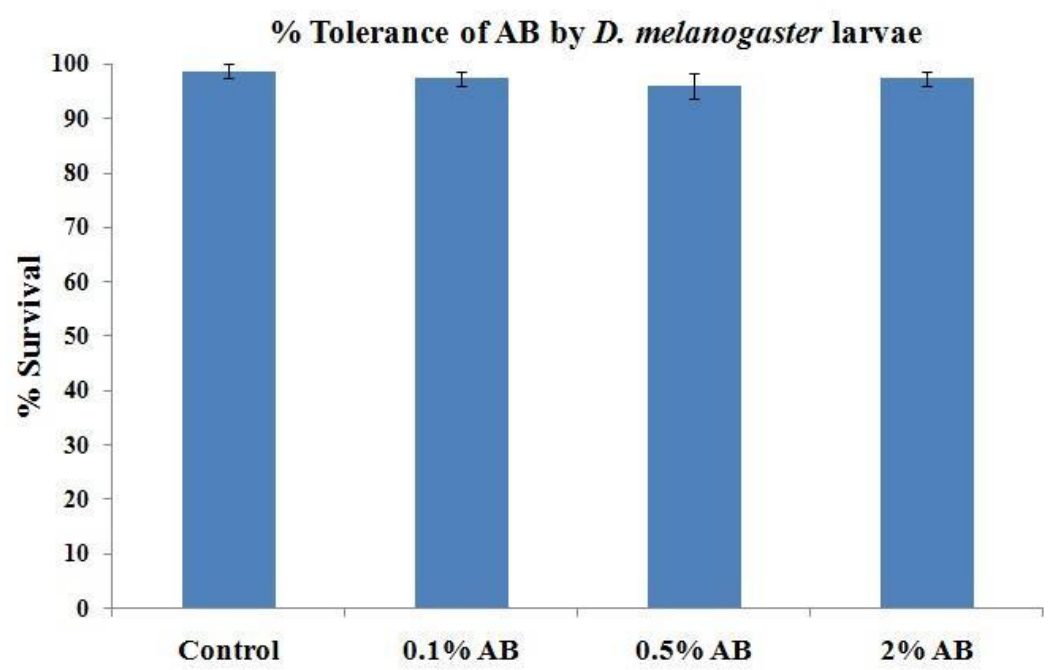

Figure 4:-D. melanogaster larvae tolerance to AB.Drosophila larvae were fed with different concentrations of $\mathrm{AB}$ and allowed to develop till adult stage. The percentage of successful adults emerged at the end was accounted for tolerance of $\mathrm{AB}$. 


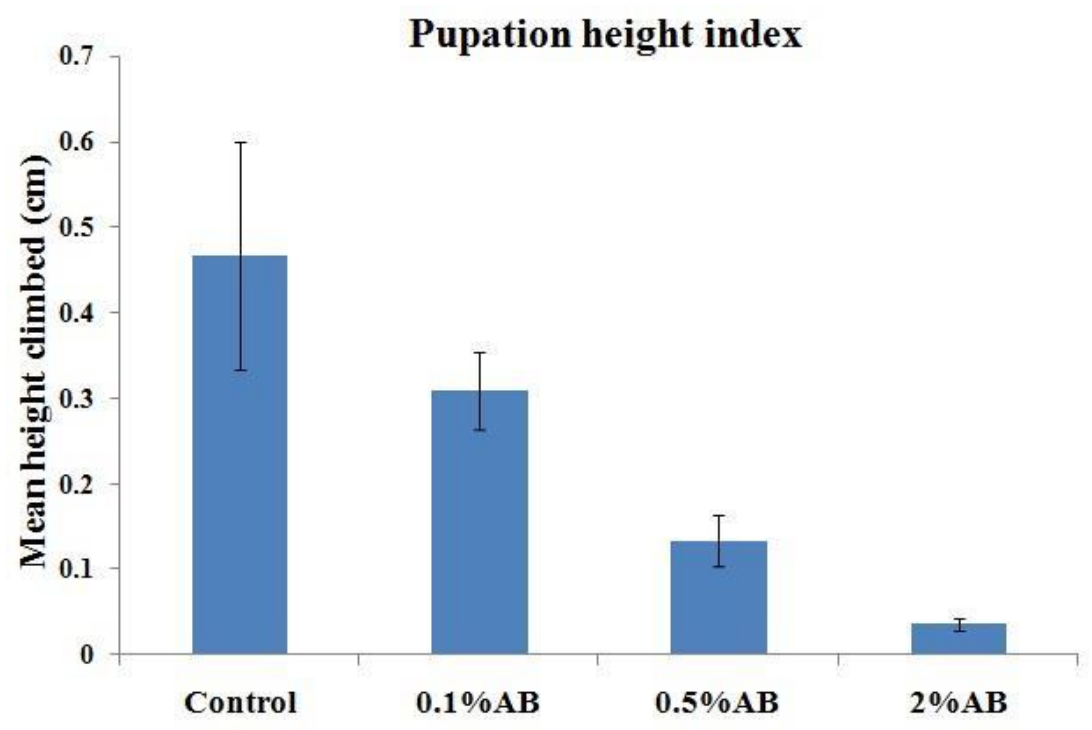

Figure 5:-Changes in Pupation height index due to the feeding of AB.The height climbed by $3^{\text {rd }}$ instar larvae away from the food source, before pupation, is represented as pupation height index. $\mathrm{AB}$ decreases the pupation height index in concentration dependent manner.

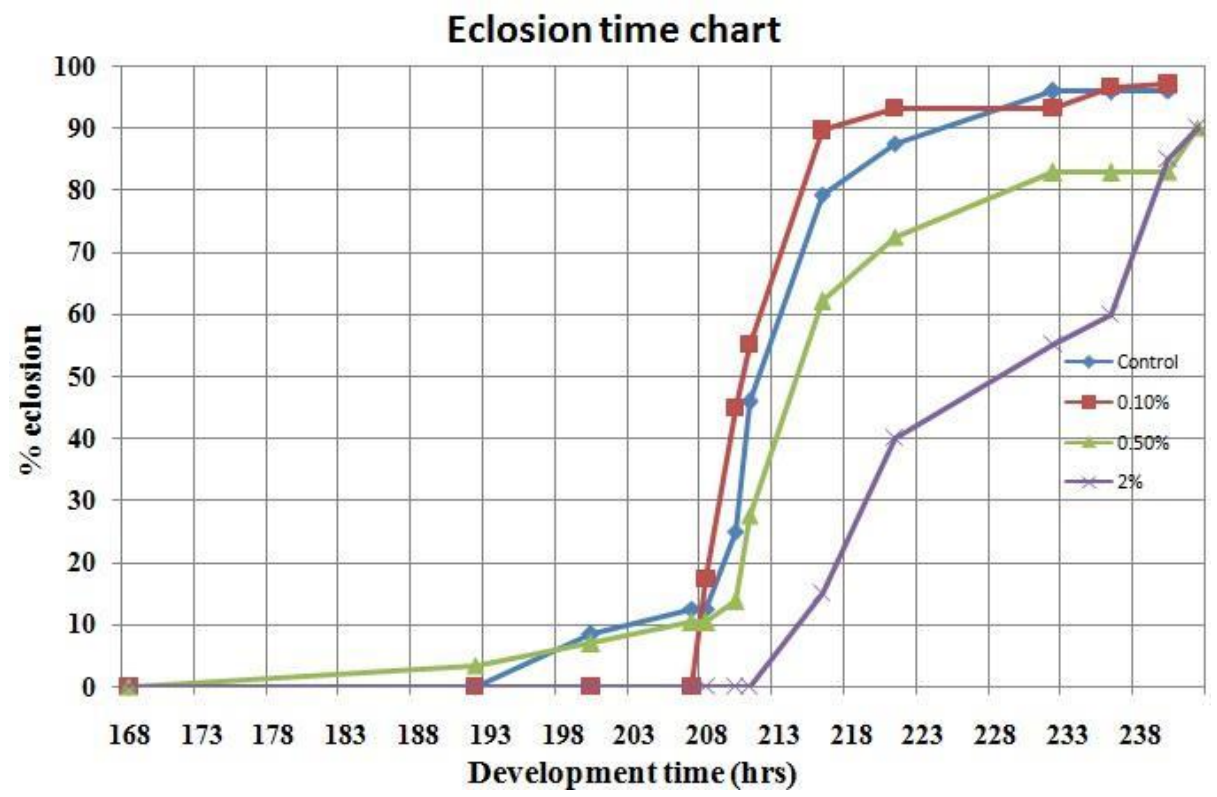

Figure 6:-Eclosion time.Pupae to adult transformation time i.e. eclosion time were monitored for $0.1 \% \mathrm{AB}$, $0.5 \% \mathrm{AB}$ and $2 \% \mathrm{AB}$ fed larvae with compared to control. 


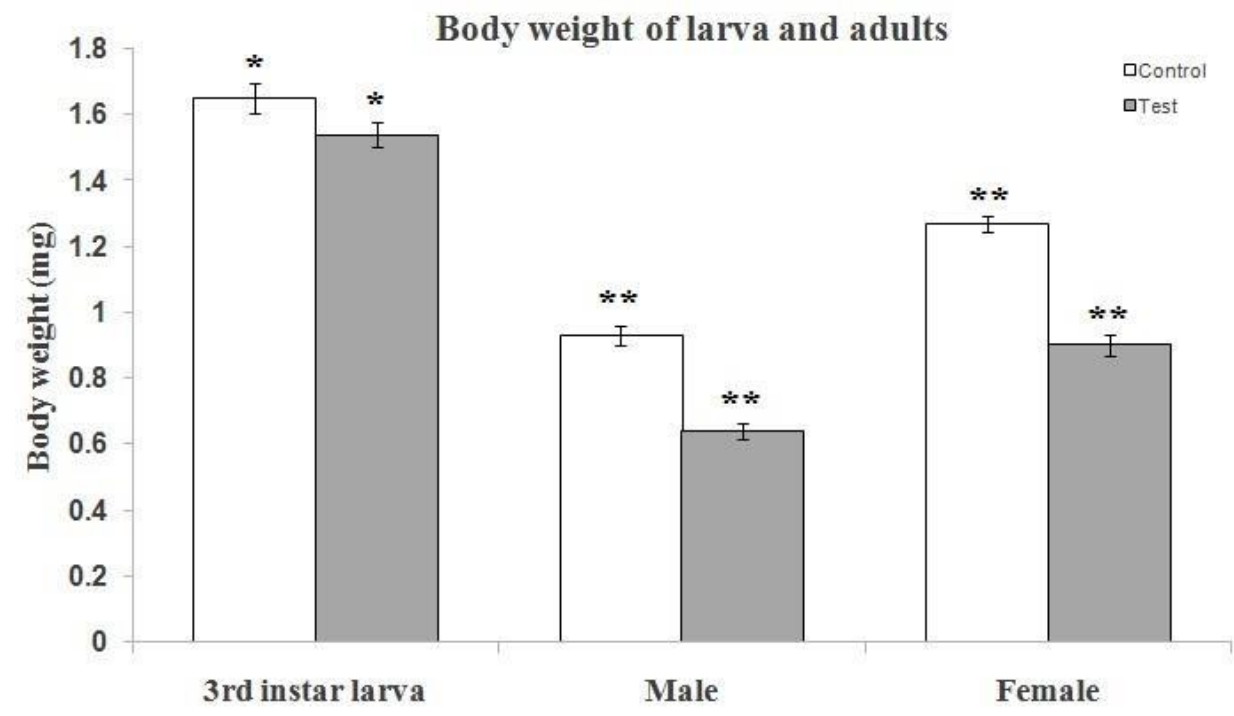

Figure 7:-Body weight analysis. Reduction in the body weight of newly emerged larvae and adults due to the feeding of $\mathrm{AB}$. ('*' represents $\mathrm{p}$-value of 0.0872 whereas '**' represents $\mathrm{p}$-value of $<0.0001$ )

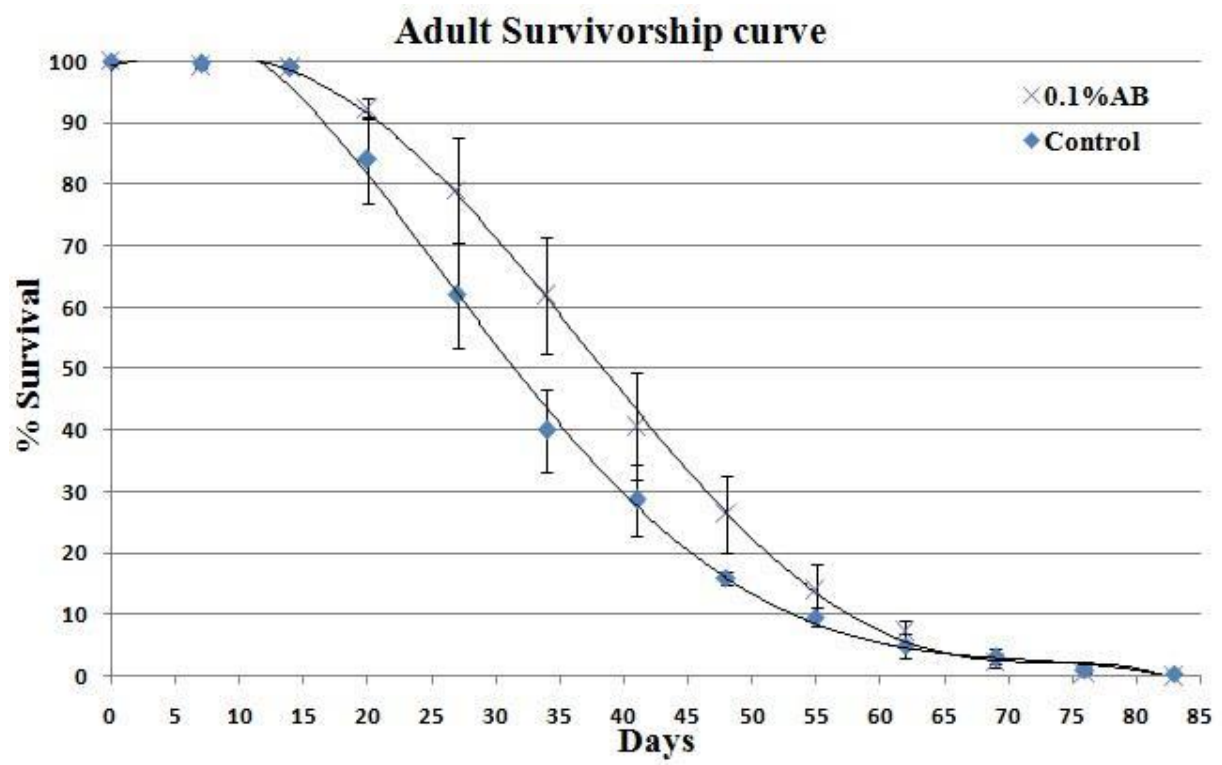

Figure 8:-Survivorship curve.The graph represents adult survivorship curve for Control and $0.1 \% \mathrm{AB}$ feeding regime where vertical bars represent standard error of the mean. 
(a)

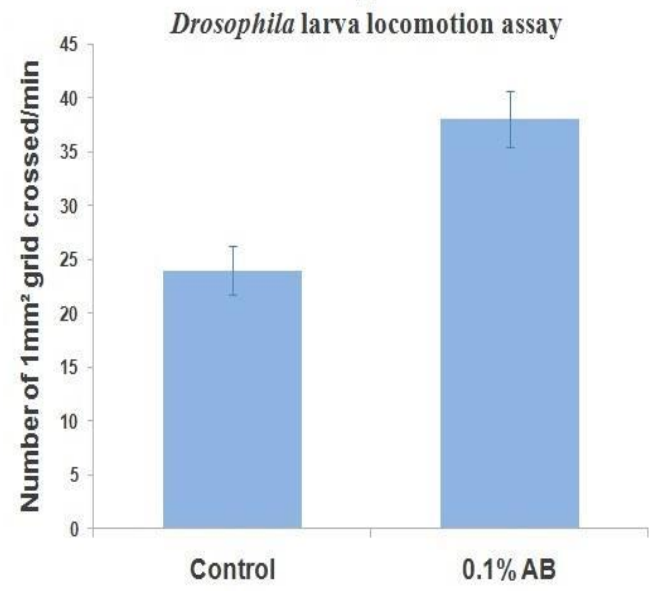

(b)

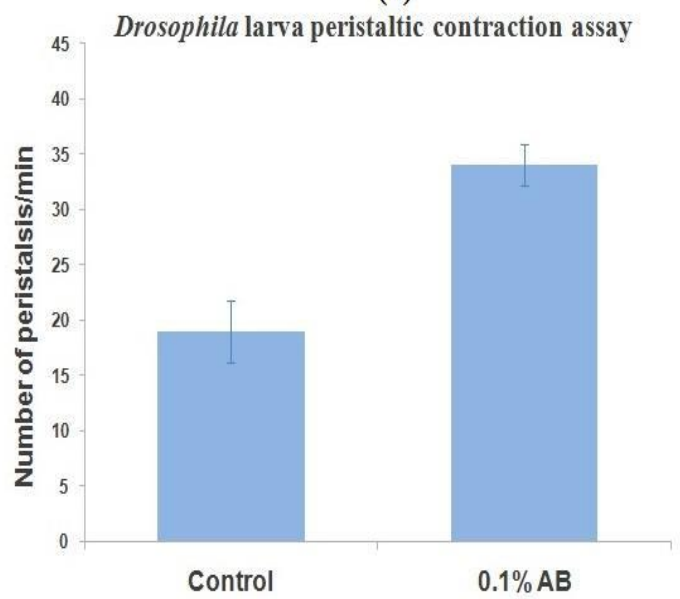

Figure 9:- (a) Locomotion assay: The number of $1 \mathrm{~mm}^{2}$ grid crossed/min by control and $0.1 \% \mathrm{AB}$ fed Drosophila larvae. (b) Peristalsis assay: The number of peristaltic contractions performed per minute by control and $0.1 \% \mathrm{AB}$ fed larvae

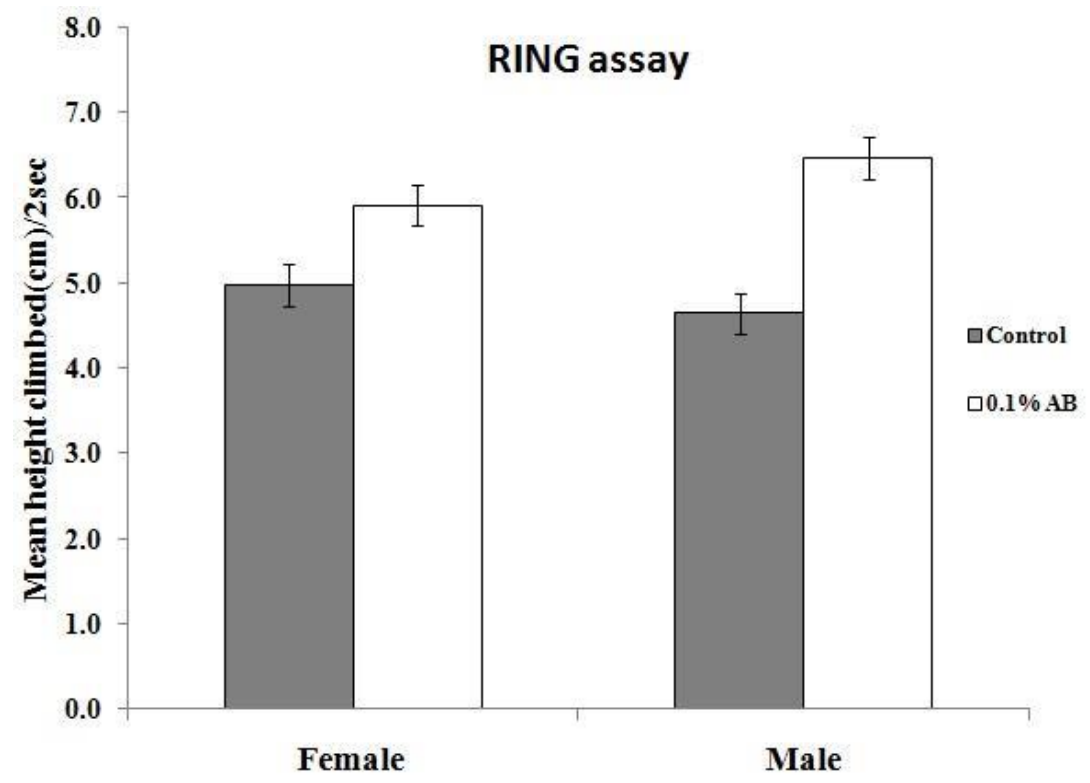

Figure 10:-RING Assay.Female and male Drosophila under the control and 0.1\%AB feeding regime tested for mean height climbed $(\mathrm{cm})$ per 2 seconds in RING assay. 
(a)

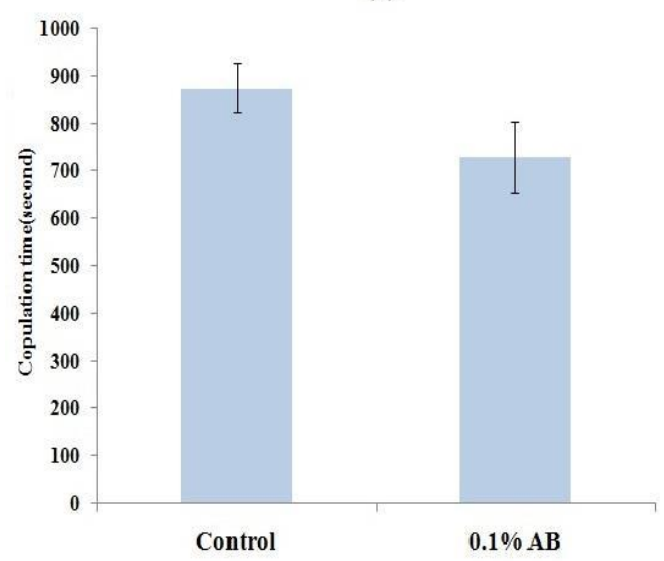

(b)

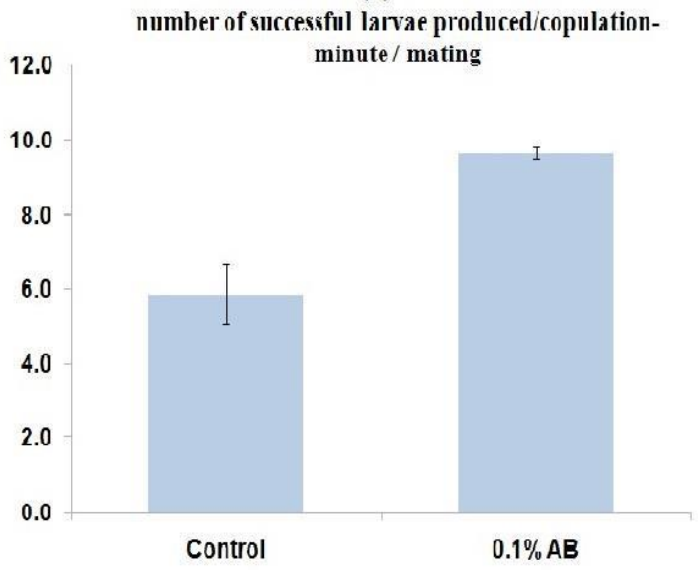

Figure 11:- (a) Copulation time: Copulation time of newly emerged Drosophila flies on control and AB feeding regime where vertical bars represents standard error of the mean. (b) Number of successful larvae produced per copulation minute per mating by adults from control and AB fed larvae

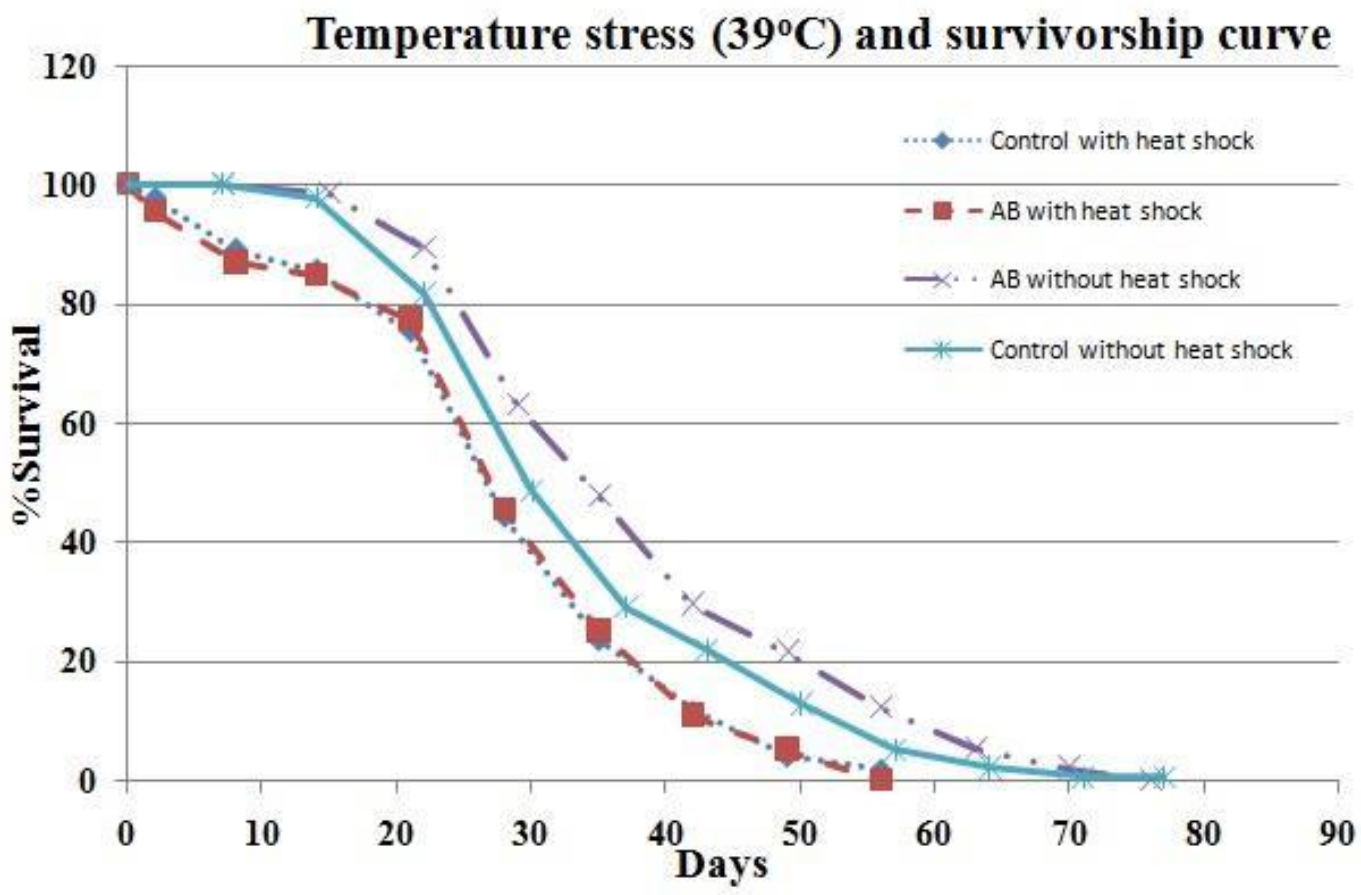

Figure 12:-Adult Drosophila survivorship exposed to $39^{\circ} \mathrm{C}$.Adults that survived the exposure of $39^{\circ} \mathrm{C}$ temperature in the heat shock experiments were further analysed for survivorship pattern represented in the graph for control and $\mathrm{AB}$ feeding regime.

\section{Discussion:-}

Ayurveda is an ancient science that dates back thousands of years. Many of the ayurvedic preparations are not validated and their exact mode of action is yet to be revealed. There is also a scarcity of studies involving the use of simple model organisms like Drosophila melanogaster for testing the safety and efficacy of the ayurvedic formulations. Some of the researchers, however, have reported an increased longevity of animal models after administration of ayurvedic preparations and modulation in different parameters of the Drosophila life cycle ${ }^{13}$.

Traditional use of $\mathrm{AB}$ in humans have been claimed to treat hepatitis, tuberculosis, asthma, etc. ${ }^{2}$ It has also been considered as an excellent cell regenerator and nerve tonic. But measuring the efficacy of $A B$ in humans and other 
mammals is cumbersome and also encompasses ethical concerns. In the present study, the effect of $\mathrm{AB}$ with respect to physiological and behavioural modifications in Drosophila has been analysed. An attempt was made to address the possibility of using certain Ayurvedic preparations to monitor the demography of Drosophila.

The various experimental designs used in this study revealed that $\mathrm{AB}$ affects physiology and behaviour of $D$. melanogaster. Food supplemented with $\mathrm{AB}$ increased the locomotor response in 3rd instar larvae, as well as the adults. As mentioned earlier, one of the common uses of $A B$ is in rejuvenation formulations and as a nervine tonic. Supporting this use, $\mathrm{AB}$ also increased the median lifespan of adult flies. It is also interesting to note that, flies fed on $0.1 \% \mathrm{AB}$ did not get any resistance from temperature shocks as compared to control. No differences were seen in percent survival of adults in any of the given temperature shock. Furthermore, the survivorship curve of adults that survived $39^{\circ} \mathrm{C}$ temperature shock for 1 hour revealed no differences in median life span.

Another important observation with the feeding of $\mathrm{AB}$ is that the time required for the development of Drosophila from egg to adult increases with an increase in the concentration of AB. This delay seems to be largely due to the delay in pupae to adult development. The factors that trigger this delay are not yet clear and more detailed investigation is required in this area.

$\mathrm{AB}$ has been found to profoundly affect the body weight of newly emerged male as well as female adults. This reduction in body weight was nearly $30 \%$. It should be noted that the duration of egg to adult development time was prolonged and this, in turn, could be largely attributed to the extended pupation period. As there is no feeding during pupation, some reduction in the body weight is presumably due to metabolism. Another plausible explanation for the reduced body weight of the adults from $0.1 \% \mathrm{AB}$ feeding regime can be the excessive water loss during extended pupation period.

The crawling assay, in combination with the peristaltic assay, can be reliably used for determining changes in locomotor functions in Drosophila early stages of developing larvae. These assays give an idea about the physical fitness of the larvae due to the given treatment. It is evident from the current study that consumption of $\mathrm{AB}$ increases the locomotor response, which in turn provides better physical fitness to the larvae. Similar to the locomotor response in larvae, the physical fitness in adults has been tested using RING assay. It was originally developed to test the locomotory response of adult Drosophila in line with negative geotaxis assay based on the performance of single flies ${ }^{10}$, who have also established that with age, RING assay performance declines in Drosophila adults. In the current study, adults from AB fed larvae have exhibited a significantly better response in RING assay which is in line with the findings in larval locomotory response. Consumption of $\mathrm{AB}$, in general, seems to cause strengthening of physical abilities in larvae and adult flies. The cellular mechanism underlying this increased efficiency is yet to be explored. It is likely that $\mathrm{AB}$ being the nanoparticle size ${ }^{14}$ gets readily incorporated in cells and tissues and enhances the antioxidant machinery ${ }^{15}$, thus boosting the physical efficiency of the organism.

Another interesting finding in this study is differences in pupation height between control and different concentrations of AB. Pupation height, or the height at which a 3rd instar larva chooses to pupate, is an important component of pre-adult development behaviour. The site selected for pupation by larvae can have a crucial role in the subsequent survival since once pupated, it remains immobile and is exposed to environmental conditions at that site $^{16,17}$. In the present study, it is observed that pupation height decreases with increase in the concentration of $A B$ in the medium. Although with high locomotor activity in $\mathrm{AB}$ fed larvae, as discussed earlier, the expectation would have been that these larvae climb higher. But this correlation is not found in the case of AB feeding. As humidity, temperature, height of culture bottle is same for all food regimes, one cannot deny the possibility of $\mathrm{AB}$ affecting the sensory system of larvae such that made it choose lower pupation height in comparison with the control larvae.

In the current study, the effect of $\mathrm{AB}$ on some of the parameters related to mating has also been studied. Though, the adults raised from $\mathrm{AB}$ fed larvae indulged less time in mating, the number of successful larvae produced per copulation minute per mating is significantly higher than in the control flies. Therefore, the role of $\mathrm{AB}$ in enhancing the fitness of sperm or fertilisation capacity cannot be ruled out as demonstrated by Babitaet al. ${ }^{6}$ in Wistar rats. However, an intensive study is still needed to validate this hypothesis. The reduction in mating time may also be the reason for increased longevity of adults derived from $\mathrm{AB}$ fed larvae, as lifespan and mating are antagonistically related, i.e. with increased mating, lifespan would decrease ${ }^{18,19}$. 
It has been observed that $\mathrm{AB}$ affects physiological and behavioural response in Drosophila larvae as well as adults. This study can be extended to numerous other ayurvedic preparations and insecticides that can alter the life expectancy and/or reproductive potential of insects. This preliminary study is an attempt to a) observe whether $\mathrm{AB}$ has any effect on Drosophila and if it does, then b)what are the parameters that can be used to monitor such changes. Some of these parameters have been explored in this study and there is a huge scope of incorporating more. Using Drosophila as a model organism, the monitoring of the demography of insects can be explored without any hindrance of ethical and experimental restrictions as it would have been in the case of most other models.

\section{Acknowledgments:-}

This work would not have been possible without the valuable support from HomiBhabha Centre for Science Education (HBCSE) - Tata Institute of Fundamental Research (TIFR). We heartily thank HBCSE-TIFR for their support. We wish to thank Mr. Vikrant Ghanekar, Biology Olympiad Cell, HBCSE -TIFR for providing initial cultures of Drosophila melanogaster. We would also like to extend our gratitude to Ms.AnupamaRonad, Biology Olympiad Cell, HBCSE -TIFR for reviewing the initial draft and providing valuable suggestion for the manuscript.

\section{References:-}

1. Pal D, Sahu CK, Haldar A. (2014): Bhasma: The ancient Indian nanomedicine. J Adv Pharm Technol Res., 5, 4.

2. Mishra L. (2004): Scientific basis for Ayurvedic therapies. (CRC press LLC), 83.

3. Pal Sanjoy Kumar (2015): The Ayurvedic Bhasma: The Ancient Science of Nanomedicine.Recent Patents on Nanomedicine, $\mathbf{5}, 12$.

4. Chaudhary A. (2011): Ayurvedic bhasma: nanomedicine of ancient India--its global contemporary perspective. J Biomed Nanotechnol., 7, 68.

5. Rajput D, Patgiri BJ, Galib R, Prajapati PK. (2013): Anti-diabetic formulations of Nāga bhasma (lead calx): A brief review.AncSci Life., 33, 52.

6. Bhatia Babita, Kale PurushottamG \&DaooJayashree V. (2013)a: The effects of abhraka bhasma on serum testosterone levels and epididymal sperm quality in heat-stroke wistar rats. International Journal of Medicine and Pharmaceutical Science, 3, 51.

7. Buwa S, Patil S, Kulkarni PH, Kanase A. (2001): Hepatoprotective action of abhrak bhasma, an ayurvedic drug in albino rats against hepatitis induced by CCl4. Indian J Exp Biol., 39, 1022.

8. Wijenayake A, Pitawala A, Bandara R, Abayasekara C. (2014): The role of herbometallic preparations in traditional medicine- a review on mica drug processing and pharmaceutical applications. $J$ Ethnopharmacol., 155, 1001.

9. Bhatia Babita, Kale Purushottam, DaooJayashree, and PanchalPranali (2012): Abhraka Bhasma treatment ameliorates proliferation of germinal epithelium after heat exposure in rats. AncSci Life., 31, 171.

10. Nichols C.D., Becnel J., Pandey U.B. (2012): Methods to Assay Drosophila Behavior. J. Vis. Exp., 61, 3791.

11. Gargano JW, Martin I, Bhandari P, Grotewiel MS. (2005): Rapid iterative negative geotaxis (RING): a new method for assessing age-related locomotor decline in Drosophila. ExpGerontol., 40, 386.

12. Frank W. Avila, Alexandra L. Mattei, Mariana F. Wolfner (2015): Sex peptide receptor is required for the release of stored sperm by mated Drosophila melanogaster females. Journal of Insect Physiology, 76, 1.

13. DwivediVibha, Anandan E. M., Mony Rajesh S., Muraleedharan T. S., Valiathan M. S., MutsuddiMousumi, LakhotiaSubhash C. (2012): In Vivo Effects Of Traditional Ayurvedic Formulations in Drosophila melanogaster Model Relate with Therapeutic Applications. PLoS ONE, 7, e37113.

14. Choube D. (2000): Brihat rasa raja sundar, uparasaprakarana. ChoukambhaOrientalia, 3, 135.

15. Bhatia Babita, Kale Purushottam, DaooJayashree (2013)b: Action of Abhraka Bhasma on Hemato-Biochemical Profile of Hyperthermic Male Wistar Rats. Int. J. Pharm. Sci. Rev. Res., 19, 47.

16. Sameoto D. D. \& Miller R. S. (1968): Selection of pupation site by D. melanogaster and D. simulans. Ecology, 49, 177.

17. Wallace B. (1974): Studies on intra- and interspecific competition in Drosophila. Ecology, 55, 227.

18. Fowler K, Partridge L. (1989): A cost of mating in female fruit flies. Nature, 338, 760.

19. Rice WR. (1996): Sexually antagonistic male adaptation triggered by experimental arrest of female evolution. Nature, 381, 232. 